\title{
Exploring 'generative mechanisms' of the antiretroviral adherence club intervention using the realist approach: a scoping review of research-based antiretroviral treatment adherence theories
}

\author{
Ferdinand C. Mukumbang ${ }^{1,2^{*}}$, Sara Van Belle $e^{2,3}$, Bruno Marchal ${ }^{1,2}$ and Brian van Wyk
}

\begin{abstract}
Background: Poor retention in care and non-adherence to antiretroviral therapy (ART) continue to undermine the success of HIV treatment and care programmes across the world. There is a growing recognition that multifaceted interventions - application of two or more adherence-enhancing strategies - may be useful to improve ART adherence and retention in care among people living with HIV/AIDS. Empirical evidence shows that multifaceted interventions produce better results than interventions based on a singular perspective. Nevertheless, the bundle of mechanisms by which multifaceted interventions promote ART adherence are poorly understood. In this paper, we reviewed theories on ART adherence to identify candidate/potential mechanisms by which the adherence club intervention works.
\end{abstract}

Methods: We searched five electronic databases (PubMed, EBSCOhost, CINAHL, PsycARTICLES and Google Scholar) using Medical Subject Headings (MeSH) terms. A manual search of citations from the reference list of the studies identified from the electronic databases was also done. Twenty-six articles that adopted a theory-guided inquiry of antiretroviral adherence behaviour were included for the review. Eleven cognitive and behavioural theories underpinning these studies were explored. We examined each theory for possible 'generative causality' using the realist evaluation heuristic (Context-Mechanism-Outcome) configuration, then, we selected candidate mechanisms thematically.

Results: We identified three major sets of theories: Information-Motivation-Behaviour, Social Action Theory and Health Behaviour Model, which explain ART adherence. Although they show potential in explaining adherence bebahiours, they fall short in explaining exactly why and how the various elements they outline combine to explain positive or negative outcomes. Candidate mechanisms indentified were motivation, self-efficacy, perceived social support, empowerment, perceived threat, perceived benefits and perceived barriers. Although these candidate mechanisms have been distilled from theories employed to explore adherence to ART in various studies, the theories by themselves do not provide an explanatory model of adherence based on the realist logic.

\footnotetext{
* Correspondence: mukumbang@gmail.com

'School of Public Health, University of the Western Cape, Cape Town, South

Africa

2Department of Public Health, Institute of Tropical Medicine, Antwerp,

Belgium

Full list of author information is available at the end of the article
} 
(Continued from previous page)

Conclusions: The identified theories and candidate mechanisms offer possible generative mechanisms to explain how and why patients adhere (or not) to antiretroviral therapy. The study provides crucial insights to understanding how and why multifaceted adherence-enhancing interventions work (or not). These findings have implications for eliciting programme theories of group-based adherence interventions such as the adherence club intervention.

Keywords: Adherence, Antiretroviral therapy, Generative mechanism, Realist evaluation, Retention in care

\section{Introduction}

HIV/AIDS remains a major problem in many regions of the world, especially in Sub-Saharan Africa (SSA). Since the inception of the global response in the early 1980s, major progress has been made towards fighting the epidemic. Through the rapid rollout of antiretroviral therapy (ART), at least 15 million people were accessing ART by 2015 globally [1]. However, most health systems in SSA face numerous new challenges emanating from scaling-up ART for people living with HIV/AIDS (PLWHA). Prominent among these challenges are sub-optimal adherence to ART, poor retention in care, and congestion of the primary health care (PHC) facilities [2].

Some solutions to addressing these challenges have been provided through the design and implementation of various HIV treatment and care models. In most cases, these models are designed to operate parallel from mainstream ART care delivery, thus known as differentiated models. These differentiated models streamline HIV treatment and care and adapt the care components to the needs of the targeted group. Results from evaluation studies show that models of differentiated care have the potential to address issues of sub-optimal adherence to ART, poor retention in care and to decongest the PHC facilities [3]. Therefore, differentiated HIV treatment and care is increasingly acknowledged as an essential approach to improving ART programmes and service delivery.

\section{Background}

Increased universal access and improved effectiveness of antiretroviral drugs have re-defined the HIV epidemic from a deadly infectious disease to a chronic disease [4]. Thus, the health care needs of ART patients resemble those of people with chronic non-communicable diseases [5]. As a result, the treatment and management of HIV conform to the management of chronic diseases, which focuses principally on adherence to medication, retention in care and management of co-morbidities. Consequently, HIV treatment and care are faced with similar challenges of poor retention in care and suboptimal adherence to medication.

Optimal long-term adherence $(>95 \%)$ is seldom achieved, although perfect adherence $(100 \%)$ is recommended for patients on ART. It is observed that most PLWHA show periods of high-level adherence followed by periods of low-level adherence [6]. Moreover, adherence rates tend to decrease as patients' time on ART increases [7]. Although earlier studies suggested that a $95 \%$ or more adherence rate to HIV medication was required to achieve medication effectiveness, with the advent of more potent regimens, a moderate adherence (75\%) can still produce the required viral suppression without producing drugresistant mutations [8]. Nevertheless, some patients still fail to maintain moderate adherence to ART for various reasons.

Adherence to ART is complex and dynamic [9]. Adherence (with regard to ART) entails following the treatment plan, taking medications as prescribed (times and frequencies), and following instructions regarding food and other medications [10, 11]. The focus of this definition is on medication use and it highlights the notion of patients conforming to the recommendations of the care providers [4]. To achieve sustained use of ART and therapeutic goals, the patient is required to adopt an effective self-management strategy, maintain contact with the health system to ensure continued, uninterrupted drug supply, and use other important monitoring services such as CD4 count and viral load measurement.

Achieving a sustained engagement under the care umbrella is key to obtaining good clinical outcomes for patients on ART [10]. The concept of sustained engagement or retention in care relates to the ability of patients to adhere to critical aspects of care, such as attending regular follow-up appointments, doing scheduled lab tests, and other monitoring activities [11]. The World Health Organization [12] defines retention in care as "the engagement in a comprehensive package of prevention, support and care services irrespective of the particular clinic site." For patients who are on ART, therefore, remaining in care offers the opportunity to receive their ARV medication without interruption, of being assessed for possible medication toxicities, of being managed of side effects and for detecting treatment failure as soon as it occurs to take the necessary action [13]. In addition, being retained in care should provide patients with access to psychosocial support and secondary prevention messages that can guide the patients towards optimising self-management of their lifelong condition. 
Poor retention in HIV care and adherence behaviours pose major challenges to the effectiveness of ART. Adhering to medication and remaining in care have the potential to improve the quality of life of patients and prevent further spread of the HIV infection. Various studies have explored the barriers and facilitators to adherence and retention in care for patients on ART [14-16]. As Table 1 illustrates, a large number of factors have been identified.

There is currently a consensus that to address issues of non-adherence and suboptimal retention in care, a variety of ART adherence support interventions has to be conceived and implemented. These interventions range from individual-level interventions to relational (patient-provider relationships such as continuity of care) to health systems interventions (e.g. task-shifting and medication distribution systems). Interventions for improving retention in care and adherence at the individual and relational level and for which there is evidence include psychosocial assessment and treatment, medication adherence counselling, home visits/a buddy system, directly observed therapy, reminder systems through Short Message Service (SMS), improving clinic accessibility, and social support [17]. Table 2 presents various adherence intervention types to improve adherence among patients on ART.

Although theories have been employed by researchers to understand how each of these single ART adherence support interventions (e.g. motivational counselling) work at a certain point in time, the long-term effectiveness of these interventions has not been established. According to Simoni et al. [18], the effects of each of these adherence-enhancing interventions targeting the affective, cognitive, behavioural or biological aspects are usually small to modest and tend to fade over time. To achieve effective and sustained effects, community-based ART models employing a combination of patient-related, relational and health system-related interventions have been proposed [19]. According to the World Health Organization [20], multifaceted - use of two or more adherence-enhancing strategies - interventions should consider "a combination of actively involving patients in their own healthcare decisions, provision of appropriate support, multidimensional educational programmes that teach behavioural skills to the patient to enhance his or her adherence, and tailoring of the regimen to fit the patient." Examples of such interventions include the ART adherence club intervention in Western Cape Province of South Africa [21], the community adherence group in Tete, Mozambique [22] and Lesotho [23] and the medication adherence club in Nairobi, Kenya [24]. These interventions have shown better results with regard to the retention of patients in ART care and adherence to ART compared to the standard care operated within the various health facility settings [25-28].

The evidence of the effectiveness of single interventions targeting the behavioural, cognitive, affective or biological aspects of patients on ART is mixed [28]. A review of qualitative studies conducted by WHO revealed that factors such as stigma and discrimination undermine the success of single ART adherence-enhancing interventions [29]. It has been demonstrated that the effectiveness of these interventions could be enhanced "when delivered in a way that resonates with local cultural norms, religious

Table 1 Barriers/facilitators to adherence and retention in ART care in Sub-Saharan Africa

\begin{tabular}{|c|c|c|}
\hline Category & Barriers & Facilitators \\
\hline Individual-Related Factors & $\begin{array}{l}\text { - Age (being younger) } \\
\text { - Depression (Mental health) } \\
\text { - Forgetfulness } \\
\text { - Substance abuse } \\
\text { - Poor self-efficacy } \\
\text { - Low Health literacy } \\
\text { - Perceived wellness }\end{array}$ & $\begin{array}{l}\text { - Age (being older) } \\
\text { - Good self-efficacy } \\
\text { - Good health literacy } \\
\text { - Constructive Health beliefs }\end{array}$ \\
\hline Medication-Related Factors & $\begin{array}{l}\text { - Medication side effects } \\
\text { - Medication dosing (Complex regimen) } \\
\text { - Treatment fatigue }\end{array}$ & $\begin{array}{l}\text { - Simple drug regimen } \\
\text { - Simple dosing } \\
\text { - Use of mechanical devices and technologies }\end{array}$ \\
\hline Health System Factors & $\begin{array}{l}\text { - Access to ART (Medication stock outs) } \\
\text { - Relationship with health care providers } \\
\text { - Staff shortages } \\
\text { - Long waiting times } \\
\text { - Poor services delivery }\end{array}$ & $\begin{array}{l}\text { - Adequate availability of human resources } \\
\text { - Adequate availability of resources } \\
\text { - Good relationship with health care providers }\end{array}$ \\
\hline Socio-economic Factors & $\begin{array}{l}\text { - Poverty } \\
\text { - Lack of family support } \\
\text { - Food insecurity } \\
\text { - Stigma and discrimination } \\
\text { - Transportation challenges }\end{array}$ & $\begin{array}{l}\text { - Short distances and reduced transport fees } \\
\text { - Financial viability }\end{array}$ \\
\hline Socio-cultural Factors & $\begin{array}{l}\text { - Alternative treatment } \\
\text { - Male dominance and gender-based violence } \\
\text { - Religious beliefs }\end{array}$ & $\begin{array}{l}\text { - Beneficial socio-cultural practices } \\
\text { - Social support }\end{array}$ \\
\hline
\end{tabular}


Table 2 Types of interventions to improve adherence [18]

\begin{tabular}{lll}
\hline Intervention level & Intervention & Intervention type \\
\hline Individual & - Adherence monitoring & - Behavioural \\
& - Directly observed therapy & - Behavioural \\
& - Reminder systems (SMS) & - Behavioural \\
& - Pre-treatment education & - Cognitive \\
& - Counselling (motivational & - Cognitive \\
& counselling) & - Biological \\
& - Food parcels & \\
Relational & - Peer support & - Affective \\
& - Home visits & - Affective \\
& - Treatment partner/buddy & - Affective \\
& System & \\
Health system & - Task-shifting & - Structural \\
& - Simplifying medication & - Structural \\
& - Alternative distribution & - Structural \\
& - Antiretroviral chronic clubs & - Multifaceted \\
& - Community adherence groups & - Multifaceted \\
\hline
\end{tabular}

beliefs and socio-economic development" [30]. We conclude that for better benefits, multifaceted, long-term and flexible approaches that target specific barriers to adherence may offer an opportunity for success as they seek to address barriers to medication adherence at all levels while reinforcing adherence behaviours.

The evaluation of the effectiveness, efficacy and performance of multifaceted adherence-enhancing interventions has been conducted in various settings. There is evidence that these interventions produce better results with regard to improving and sustaining adherence to ART and retaining patients in care compared to various standard treatment and care schemes operative in the various contexts [31]. Recently, there is an impetus to evaluate such programmes, not only to inform their efficacy and the effectiveness, but evaluators are encouraged to also unearth the "programme theory" [32] - a theory, model or set of assumptions of how and why an intervention contributes to a set of specific outcomes [33] - that underpin these interventions.

This study is nested within a larger project, "Realist evaluation of the antiretroviral treatment adherence club programme in selected primary health care facilities in the metropolitan area of Western Cape Province, South Africa" [34]. The first step of this realist evaluation is to elicit an initial programme theory of the adherence club intervention. To start, we explored the perspectives and assumptions of the programme designers and managers [35]. We then reviewed the literature on how similar interventions that have been implemented work, why they work, for whom they work and under what circumstances. In this paper, we report on a scoping review of various (social science/psychological/behavioural) theories applied to explain adherence to ART. The aim was to identify and assess the use of theories in the adherence literature and to see whether we could draw additional mechanisms from that literature, which could help us to refine the initial programme theory that will be empirically tested.

\section{Understanding 'mechanism' in realist evaluation}

Realist evaluation is a member of the family of theorybased evaluation approaches [36]. It operationalises the causal mechanisms that are likely to operate for a programme to work and the contexts in which these mechanisms are triggered to lead to the outcomes. Realist evaluation begins by eliciting an initial programme theory, which is subsequently tested, often by employing qualitative and quantitative research methods. The initial programme theory is revised based on the empirical findings and can lead to a middle range theory that explains in which contexts the intervention works by triggering specific mechanisms. Realist studies do not only answer the question 'what works?' but 'how or why does this work, for whom, in which circumstances?' [36].

While conducting a realist evaluation, identifying the 'generative mechanism' - the underlying social drivers of behaviour - is key, as it is one of the central elements of its explanation strategy. Pawson and Tilley explain that when programmes are implemented, they provide a resource, an opportunity or a constraint of some kind that influences the target person's decision-making [36]. Therefore, a generative mechanism is "the process of how subjects interpret and act upon the intervention (or components of the intervention)" [37]. Lacouture and colleagues offered another description of 'mechanism' from a public health perspective: "a mechanism is hidden but real. It is an element of reasoning and reaction of agents in regard to the resources available in a given context to bring about changes [outcome] through the implementation of an intervention and evolves in an open space, time and open system or relationships" [38]. Based on these definitions, three main characteristics of mechanisms can be identified: (1) they are usually invisible; (2) sensitive to variations in context, and (3) generate outcomes [39].

The other two components required to complete theory building in realist evaluation are context (of action) and outcome [40]. According to generative causality in realist evaluation, a generative mechanism can only be triggered in specific context conditions to cause the expected outcome. This causal relationship is represented thus: an outcome $(\mathrm{O})$ is generated by a mechanism $(\mathrm{M})$ being triggered in a specific context (C). In realist evaluation, this relationship is explored using the contextmechanism-outcome (CMO) configuration heuristic [36, 41]. To facilitate understanding, the intervention (I) and the actors involved in the intervention (A) are also being represented, leading to the Intervention-Context-ActorMechanism-Outcome (ICAMO) configuration [42]. 
From our previous work on framing the concepts of ART adherence and retention in care using realist logic, we developed the framework presented in Fig. 1. This framework illustrates that the resources (information, skills, material resources, support) provided by the adherence club intervention, works through mechanism(s) acting through the actors (patients, lay counsellors and clinicians), which [the mechanisms] are sensitive to various contexts (distal and proximal), to cause the intended outcomes (adherence to medication and retention in care). Our conceptualisation considered both psychological and relational (social) mechanisms. Examples of psychological mechanisms include motivation, self-efficacy and empowerment as these emerge from an individual's cognition. The relational mechanisms were considered with regard to the relationship that the patient shares with the care providers (e.g. trusting relationship and social support) and fellow group members (group dynamics and culture that exist within each group). These are in essence relational mechanisms that causally affect people by virtue of the individual's position relative to his/ her social relationships. Therefore, with regard to groupbased adherence models, mechanisms can play out at the level of individuals, dyadic relations (between provider and patient, for instance) and/or the group.

\section{Methods}

We carried out a scoping review [43], with a narrative integration of the relevant evidence [44]. The approach allows for the identification and review of theories and concepts and for summarising empirical and theoretical literature to develop a comprehensive picture of a particular phenomenon. We aimed at reviewing, critiquing and synthesising the literature on theories that are used to explain how adherence-enhancing interventions work or are expected to work. The narrative integration of the relevant evidence holds the potential to integrate the literature on these theories into frameworks as well as generate new perspectives to develop a meta-theory the means of conceptual exploration - across theoretical domains [45].

Conducting a scoping review following the guidance of Arksey and O'Malley [43] suited our purpose because our focus was not primarily on the study designs and the findings that the studies presented, but rather on the theories that guided the conceptualisation of the studies. For this reason, there was no need to conduct a methodological appraisal of the studies that were included in the review. In conducting the review, we followed the five iterative steps: (1) Problem identification; (2) Literature search; (3) Data collection: Extraction of data from selected literature; (4) Collate, summarise and report the results; (5) Discussion of results.

\section{Problem identification}

Various reviews have been conducted to identify and investigate the roles of health behaviour change theories and health psychology theories in developing strategies to improve HIV/AIDS medication adherence [46]. With

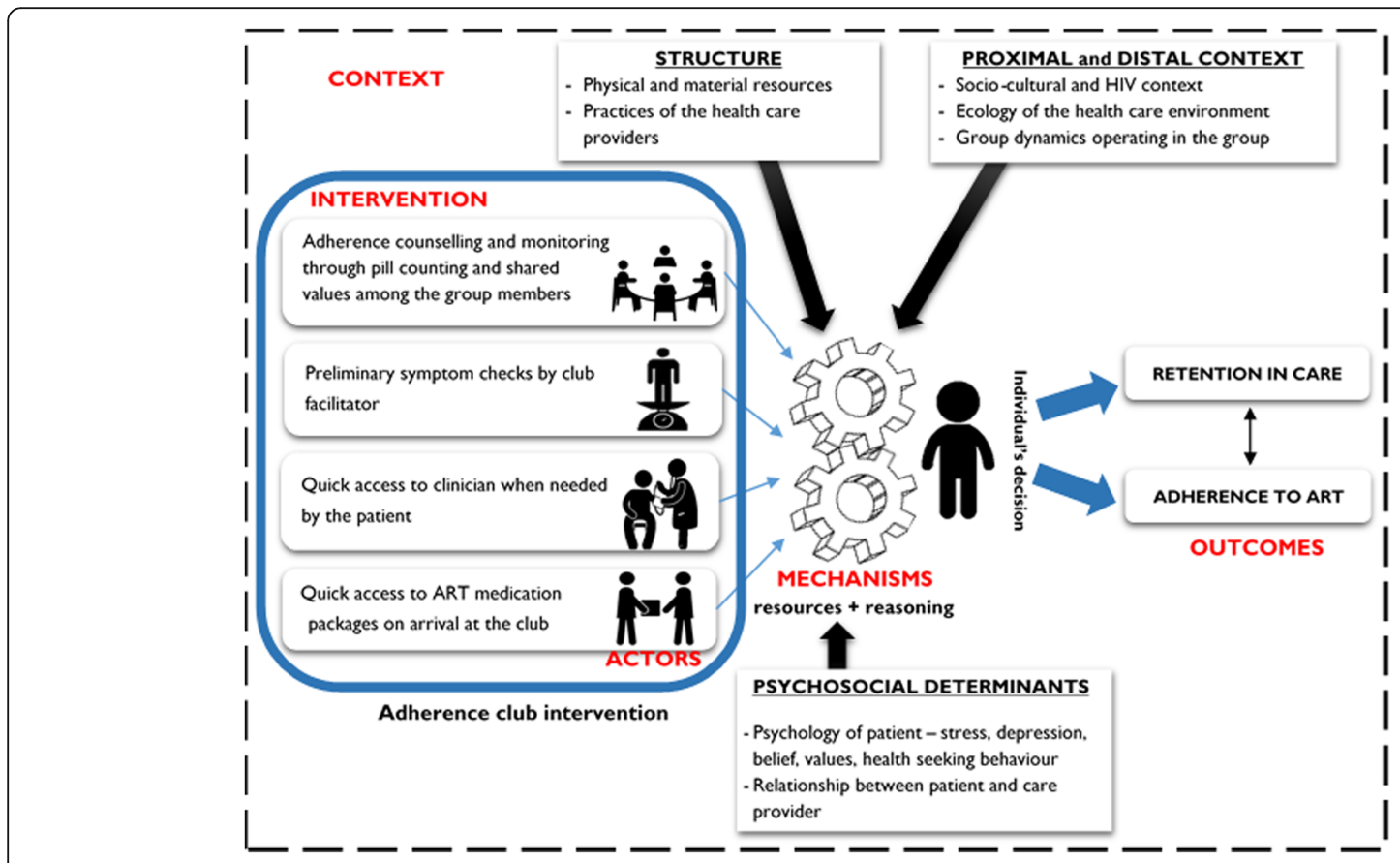

Fig. 1 An intervention-context-actor-mechanism-outcome (ICAMO) framework representing how and why the adherence club intervention works (Source: Authors') 
this review, we aimed at searching for research-based (peer-reviewed) application of behaviour change theories to provide explanations of how adherence-enhancing interventions work, with the overall goal of developing an initial programme theory of a group-based adherence intervention - the adherence club intervention.

\section{Literature search}

The first author and a research assistant searched for articles for possible inclusion in the review. We applied two methods to search for papers: electronic database searching (PubMed, EBSCOhost, CINAHL, PsycARTICLES and Google Scholar) and manual reference list search. We searched each database using the following Medical Subject Headings (MeSH) search phrases "Adherence [OR] compliance to antiretroviral therapy [AND] theories [OR] models." We also searched the reference list of the papers identified through the database search. We defined the inclusion criteria for the review using the PICOT mnemonics for reviews.

- Patient population: Adult (18+ years) patients on ART

- Intervention or Interest area: Adherence (compliance) theories or models

- Comparison interventions: Depends on study

- Outcome: ART adherence and retention in care

- Time: 2000-2015

We excluded non-English papers. We assessed the search hits and selected only the articles with relevant titles. We stopped searching for articles when we reached saturation, i.e. when no new papers were identified. Table 3 below illustrates the data search process and the final number of studies that were retained for review.

The screening of the 84 articles was conducted by the first author and the research assistant and proceeded in three stages: (1) screening by title, (2) screening by abstract, and (3) reading the full article. Twenty-six (26) articles were obtained for the inclusion in the review process. Figure 2 outlines the data screening and selection process following the Preferred Reporting Items for
Systematic Reviews and Meta-Analyses (PRISMA) flow diagram protocol.

\section{Data collection}

Extraction of data from the identified papers was done under the following topics: (1) Study citation and setting, (2) Study purpose and theory of interest, (3) Study design and methods, (4) Study limitations, (5) Conclusions/Recommendations. Refer to Additional file 1 for the data extraction process.

\section{Analysis}

We examined each theory to identify context, mechanism and outcome components as defined in realist terms (See Additional file 2 - 'Data code manual). We then assessed to what extent the theories could inform a generative causality using the intervention (I) leading to outcome $(\mathrm{O})$ by triggering a mechanism $(\mathrm{M})$ in context $(\mathrm{C})$ for specific actors (A) conceptualisation. Following this assessment, we identified the possible mechanisms - the underlying social drivers of behaviour - from each of the theories that showed potential to explain ART adherence in the realist sense.

\section{Results}

\section{Characteristics of sampled studies}

We summarised the characteristics of the studies under the following topics: Evidence type, research approach, study design, objective of theory inquiry, and study setting (continent). Table 4 below shows the general characteristics of the 26 studies included in the review. It should be noted that 17 out of the 22 primary research papers used a quantitative approach and only five research papers used other approaches.

\section{Identified theories}

The graph below (Fig. 3) represents the various theories that are discussed, tested or developed in the identified studies.

According to our judgement, three of the 11 theories applied to explain adherence to ART identified in the review featured conceptualisations that are consistent

Table 3 Search terms used and number of articles identified

\begin{tabular}{llll}
\hline Database & Keywords used & References identified & Selected \\
\hline Psych ARTICLES & Adherence [OR] compliance to antiretroviral therapy [AND] theories [OR] models & 25 & 7 \\
CINAHL & Adherence [OR] compliance to antiretroviral therapy [AND] theories [OR] models & 173 & 4137 \\
ScienceDirect & Adherence [OR] compliance to antiretroviral therapy [AND] theories [OR] models & 735 & 9 \\
PubMed & Adherence [OR] compliance to antiretroviral therapy [AND] theories [OR] models & 11,470 & 12 \\
Google Scholar & Adherence [OR] compliance to antiretroviral therapy [AND] theories [OR] models & 15 & 15 \\
Manual Search & Titles using related to adherence or compliance to antiretroviral therapy theories and models & 83 \\
Total & & & \\
\hline
\end{tabular}




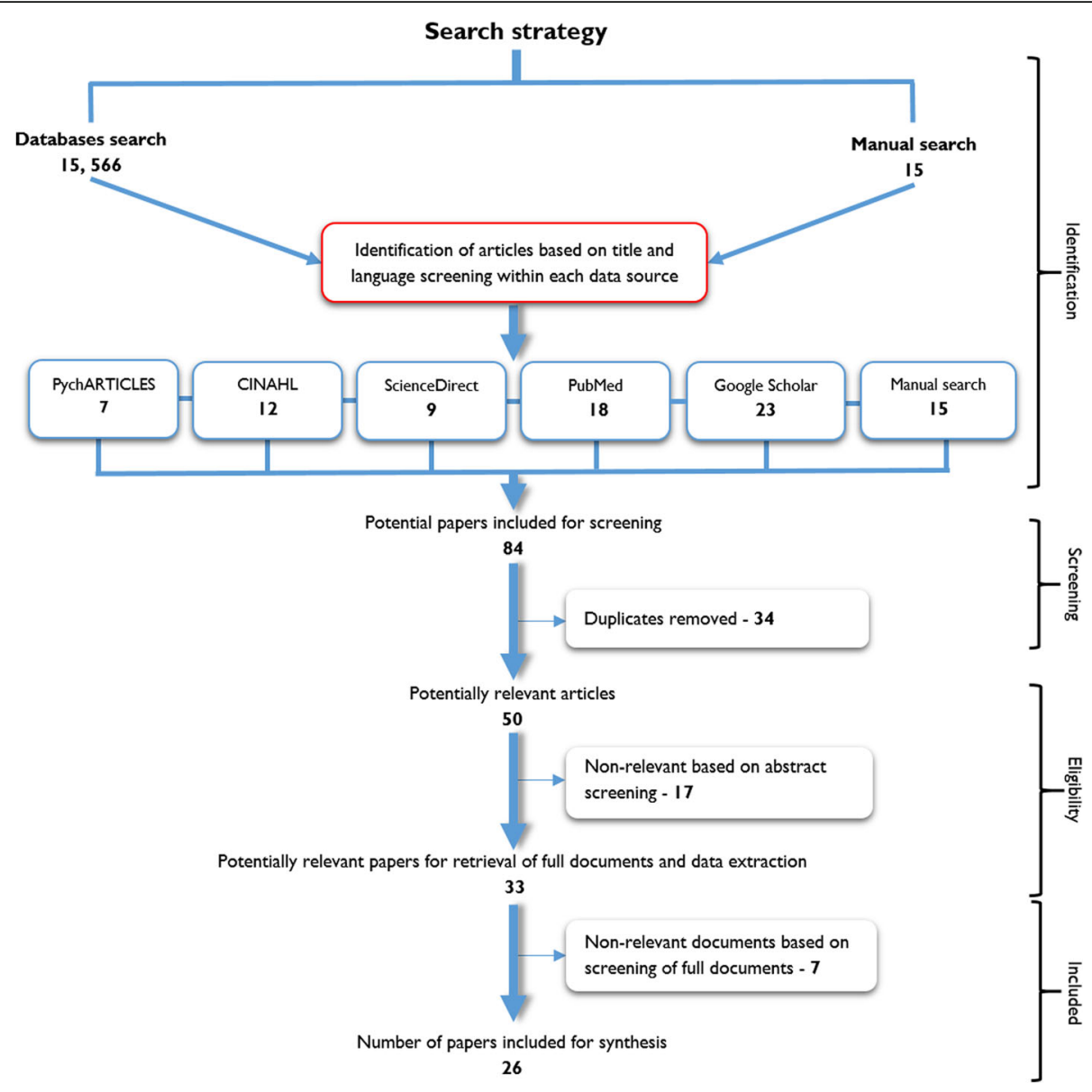

Fig. 2 Article screening process based on the PRISMA protocol

with the realist logic of causality: Information-MotivationBehaviour [47], Ewart's Social Action Theory [48] and the Health Behavioural Model [49]. In the section that follows, we look into the framing of these theories.

\section{Information-Motivation-Behaviour (IMB) model}

The Information-Motivation-Behaviour (IMB) model was discussed in 11 of the 26 articles that were included in the review. Some of the articles applied the IMB model to explain adherence to ART, or to conceptualise the factors affecting patients' adherence to ART while others used the IMB model to develop an explanation on how ART adherence occurs [50-60]. Based on the IMB model, "well-informed, well-motivated patients who possess adequate skills for enacting complex patterns of adherence-related behaviour will adhere to their ART regimen optimally over time" [57]. However, what is missing is how Intervention, Context and Mechanism interact to cause that Outcome. Figure 4 indicates how the IMB model is used to explain adherence to ART.

In Fig. 4, adherence information is (part of) the intervention that the patient receives. This information relates to the regimen, correct use of ART and the importance of adequate adherence. Additional information offered to the patients relates the side effects of the medication and possible drug interactions. According to the IMB model, this information can be a source of motivation for the patient (motivation in the psychological sense). Social support (also labelled social motivation by IMB authors) is based on how the patient perceives the support of his or her significant others. The patient's motivation (psychological or social) and self-efficacy are moderated by actor-related elements such as the psychological health of the patient (e.g. depression), unstable living situation, poor access to medical care services and substance use. The patient's motivation can be influenced by these context conditions.

According to the IMB model, adherence behavioural skills (including objective ability and perceived efficacy for performing critical skills such as acquiring selfadministering ART) play a vital role in determining whether a patient adheres to their medication or not. This notion was applicable when ART medication regimens were very complex to self-administer and required 
Table 4 Characteristics of the studies included in the review

\begin{tabular}{|c|c|}
\hline Characteristics & $N$ \\
\hline \multicolumn{2}{|l|}{ Evidence type } \\
\hline Primary research & 22 \\
\hline Review article & 2 \\
\hline Commentary & 2 \\
\hline \multicolumn{2}{|l|}{ Research approaches } \\
\hline Quantitative methods & 17 \\
\hline Qualitative methods & 5 \\
\hline Reviews & 2 \\
\hline Commentaries & 2 \\
\hline \multicolumn{2}{|l|}{ Study design } \\
\hline Cross-sectional survey & 9 \\
\hline Cross-sectional interviews & 5 \\
\hline Randomised controlled trial & 4 \\
\hline Within-subject comparison design & 1 \\
\hline Systematic review & 4 \\
\hline Elicitation (theory) design & 1 \\
\hline Participatory research & 1 \\
\hline Qualitative exploratory & 1 \\
\hline \multicolumn{2}{|l|}{ Objective of theory inquiry } \\
\hline Application of model to ART adherence & 8 \\
\hline Theory testing & 11 \\
\hline Model development & 1 \\
\hline Conceptualisation of factors into a model & 6 \\
\hline \multicolumn{2}{|l|}{ Study setting (continent) } \\
\hline Africa & 2 \\
\hline North America & 18 \\
\hline South America & 1 \\
\hline Europe & 2 \\
\hline Not applicable & 3 \\
\hline
\end{tabular}

some skills. Today, antiretroviral medication is less complicated to self-administer, as it is in most regimes, a single tablet taken once a day. The IMB model also specifies that adherence information and adherence motivation may be directly related to ART adherence in cases where medication-taking behaviours are not complex or demanding [52]. However, the IMB model is not clear about what connects each of the boxes, for instance why is it that a person with adherence behavioural skills changes his/her behaviour and becomes adherent? Neither does it present a configurational approach that builds an explanation of the outcome by linking all of the precedents in a coherent causal configuration. Therefore, the IMB model somehow remains a descriptive model of precursors and not a causal model.

\section{Ewart's Social Action Theory (SAT)}

Ewart's Social Action Theory (SAT) was explored in two studies [61, 62]. The theory focuses on behaviour change and factors such as social context and support that can assist to foster and maintain that change. According to SAT, health behaviours result from an interplay of three domains: (1) context, in the sense of the social-environmental and the specific personal attributes of the individual, (2) processes of self-change that create new or modified action scripts and (3) selfregulation as an action state (in this case adherence to medication) [62].

SAT holds that health behaviours are a result of selfchange or self-regulatory processes by which an individual makes the transition from old actions to adopting new behaviours [62]. Specific mechanisms of behaviour change that are identified in SAT include (1) problem-solving, (2) motivation, (3) generative capabilities (empowerment) and (4) social interaction processes. Remien et al., [62] suggested that the ability to problem-solve is related to social and emotional adjustments. According to SAT, patients can become motivated to adhere to their treatment based on their outcome expectancy - an

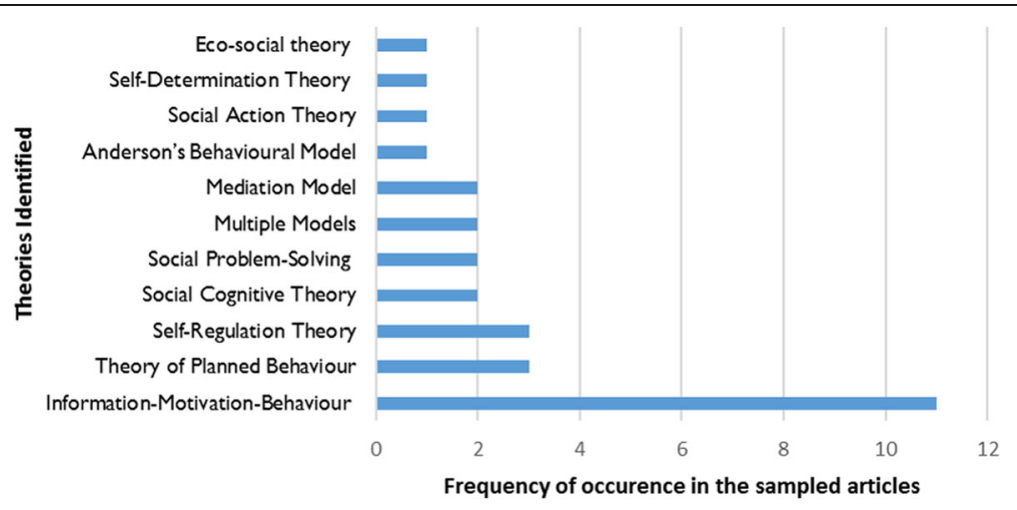

Fig. 3 A graphical representation of the theories identified in the studies included in the review and the frequency of their occurrences 


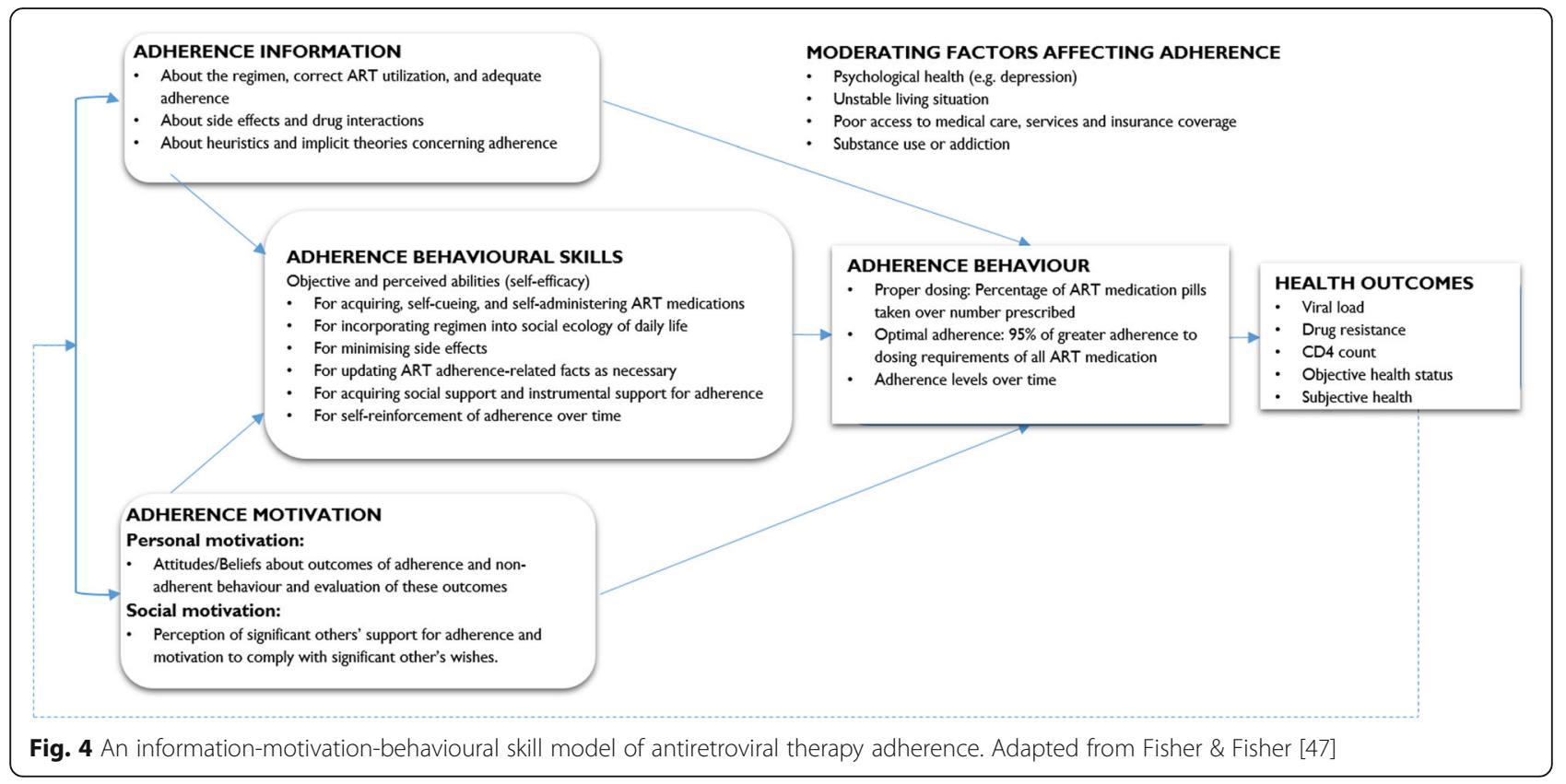

evaluation of their personal capacities to carry out the intended behaviour - as well as when they generate goals that could stimulate the expected behaviours. Empowerment as an essential generative capability refers to a sense of personal control, mastery, and power to effect change. Social interaction processes between the patient and health care providers and with other significant others are important elements in the self-change processes.

According to SAT, the intended health behaviours are shaped by broader social-environmental systems and intrapersonal factors of the individual, representing contextual influences that can either facilitate or hinder behaviour change. Physical settings and social systems both affect and interact with biological structures and processes within the individual to create intrapersonal contexts that influence goals and generative capabilities [48]. Ewart suggests that these larger social-environmental systems "contextually determine how personal change mechanisms operate" [48]. This is in accordance with the realist logic that suggests that the mechanisms can only be fired in the right contextual environment, which leads to the intended outcome. These contextual conditions according to SAT include patient's demographics, living circumstances, social network, organisational systems and physical environmental factors [62].

Ewart [48] suggests that SAT could potentially be used to specify mediating mechanisms linking organisational structures to personal health. According to Ewart, behavioural interventions (such as the adherence club intervention) strengthen self-regulatory systems in individuals that foster capacity for self-protective action. Figure 5 represents the conceptualisation of SAT to explain ART adherence.

Notably, while SAT presents a precedent of behaviour in the form of processes of self-change and stipulates that context matters, it conflates environment with personal (psychological) factors. Secondly, it does not explain which of the sub-elements of environment and selfchange are linked to which other. Therefore, although

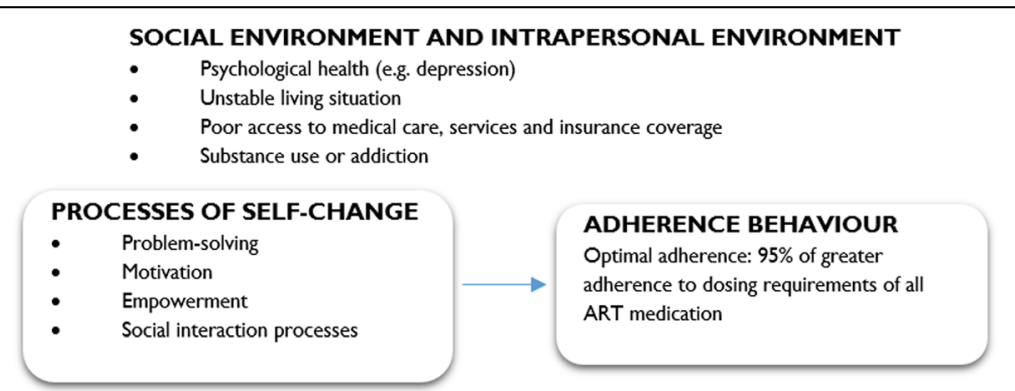

Fig. 5 Conceptualisation of the Social Action Theory for ART adherence. Obtained from Ewart [48] 
SAT presents three categories (context, mechanisms and outcomes) that can be useful to describe an adherence intervention, it falls short of explaining exactly why and how these factors combine to explain positive or negative outcomes.

\section{Health Belief Model (HBM)}

The Health Belief Model (HBM) also offers a potential explanation for adherence to ART and was explored in one study [60]. The HBM aims at assessing health behaviour of individuals through the perceptions and attitudes a person may have towards disease and negative outcomes of certain actions. The HBM was conceptualised around the individual's beliefs and attitudes captured in four constructs representing the perceived threat and net benefits [63]. These constructs are perceived susceptibility and perceived severity that make up perceived threat and perceived benefits and perceived barriers representing the net benefit [49]. In relation to ART adherence, perceived severity refers to an individual's subjective assessment of the severity of the consequences of non-adherence to ART. Perceived susceptibility refers to the individual's assessment of the personal risk of developing problems with regard to ART medication non-adherence. Perceived benefits, on the other hand, relate to an individual's assessment of the value of adhering to ART. Finally, perceived barriers relate to an individual's assessment of the obstacles to taking the medication. These constructs represent possible mechanisms that could be triggered to enforce adherence behaviour.

The 1950 model of HBM was consolidated when Becker et al. [64] published a paper that considered a range of alternative approaches to understanding the social and psychological determinants of health and illness behaviour [63]. The authors added the influence of the patient's demographic variables and psychological characteristics on the identified mechanisms. These demographic variables and psychological characteristics of the individuals are important conditions that could trigger the mechanisms identified. Finally, to complement the HBM, Becker et al. [64] included the concept of 'readiness to be concerned about health matters' which represents an individual's general health motivation. Figure 6 indicates the operationalisation of the identified concepts relevant to HBM.

When conceptualised in the context to chronic medication adherence such as ART, the HBM translates to the desire to achieve a better quality of life and the belief that adherence to ART will ameliorate the health of the patient and this would influence whether a patient adheres to their medication or not. According to Janz and Becker [49], if an individual has high perceived threat towards a disease or a health issue; low barriers to adopting healthy behaviours; and high perceived benefits to action that would help avoid the health issue; then there is an increased likelihood of the individual engaging in the recommended behaviour.

We would say that the HBM usefully describes demographic and psychological factors that influence health motivation, and more interestingly identifies the factors (candidate mechanisms) that perpetuate actual adherence behaviour. However, it also fails to operationalise a realist causal explanation. That is, it fails to identify what mechanisms or sets of mechanisms are triggered under what context of action to cause the expected outcome of interest.

\section{Discussion}

Our review identified some theories of health behaviour that have been applied to understand and enhance treatment adherence to ART and other medical treatments by a number of authors. Nevertheless, as observed by Holmes et al. [46] and Krueger et al. [65] no single theory provides

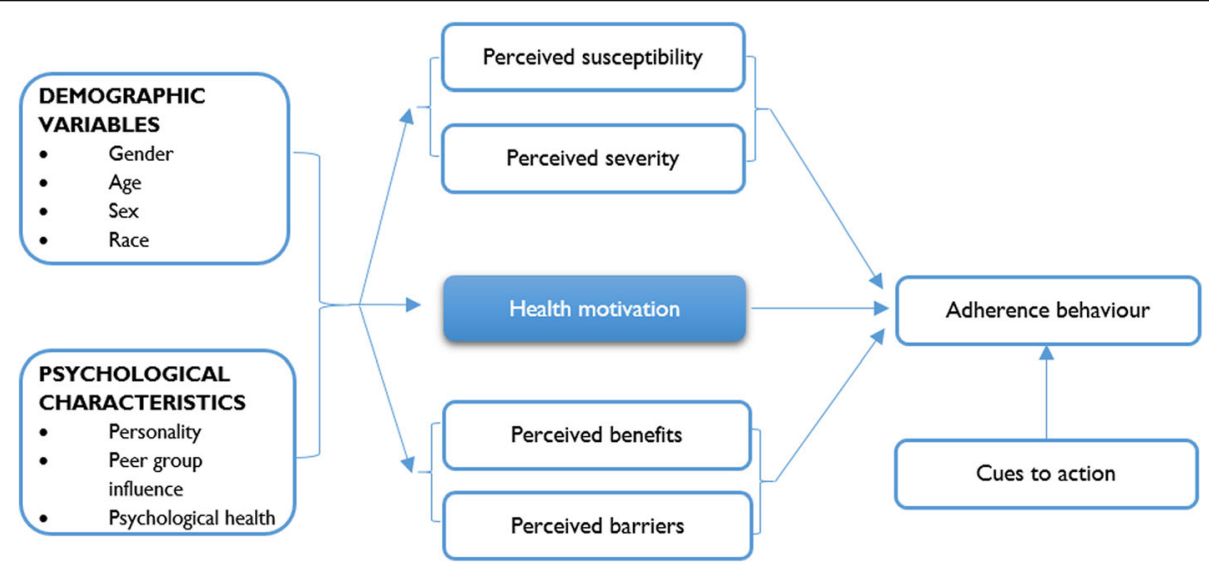

Fig. 6 Health Behavioural Model. Obtained from Munro et al. [60] 
a comprehensive picture. Our review also indicates that most studies used quantitative methods to test the theories and models, thereby adopting a variable-oriented approach to analysis to estimate the casual influence of the various variables representing the determinants. The limited qualitative papers also focused on thematically identifying barriers to adherence to medication using a theoretical framework rather than providing an explanatory model of adherence to ART using generative mechanisms.

While most of the theories applied to explain adherence identified various concepts that carry the sense of a 'mechanism' to identify and define central explanatory element(s), these concepts do not qualify or fulfil all the characteristics of a generative mechanism (social and/or psychologic drivers), that is, invisible, sensitive to variations in context and can generate outcomes. Nevertheless, these concepts could be seen as related to the set of attributes of generative mechanisms. Examples of such central elements include adherence attitude (Theory of Planned Behaviour), behavioural intention (Theory of reasoned behaviour and Theory of Planned Behaviour), problemsolving (Social Problem Solving), health belief (Health Belief Model), knowledge and behavioural skills (Selfregulatory model).

Based on our review, three theories had conceptualisations that are somewhat similar to the realist logic: IMB, HBM and SAT. Although these theories show potential to provide an explanation of how ART adherence occurs by identifying relevant concepts and indicating possible relationships and effects between these concepts, they fall short in explaining exactly why and how these concepts identified as variables - combine to explain positive or negative outcomes. In other words, they fail to show how possible mechanisms introduced by an intervention are triggered by various contextual conditions to generate the expected or observed outcome. Therefore, they fail to show generative causality. At best, these theories/models remain descriptive models of precursors and not causal models.

While a number of authors use the term 'mechanism' to identify and define central explanatory element, these concepts do not exactly qualify as mechanisms in the 'realist' sense (social and/or psychologic drivers). Possible 'mechanisms' identified from the Health Belief Model that may qualify as a mechanism in the realist sense include: perceived susceptibility, perceived severity, perceived benefits and perceived barriers. From the IMB model, possible 'mechanisms' identified were motivation and self-efficacy. From Ewart's SAT, possible mechanisms following the realist logic include empowerment, perceived social support and motivation. Although these theories identified various concepts that could possibly be conceptualised in the realist sense, in most part, they only provided descriptive models of how adherence to ART could be achieved. In this sense, they fail to provide an explanation of exactly why and how these factors and concepts combine and contribute to explaining positive or negative outcomes.

Having identified some potential mechanisms for ART adherence, the next step is to relate this information to how the adherence club intervention contributes or is expected to contribute towards generating these mechanisms and how does the contextual environment activate these mechanisms to cause the intended outcome (adherence to ART). The generative mechanisms identified in the review represent psychological mechanisms (motivation, self-efficacy, empowerment, perceived threat and benefits) and relational mechanisms (perceived social support). Bandura [66] suggested that understanding cause-effect should be regarded as a causal system in which "socio-structural influences operate through psychological mechanisms to produce behavioural effects." The generative mechanisms identified in this review will be examined and coordinated with other mechanisms and context conditions identified from an exploratory study of the opinions and assumptions on the adherence club managers and designers to elicit the initial programme theory of the adherence club intervention.

\section{Strengths and limitations of the review}

The review was limited to an extent by the fact that most of the studies did not fully describe the theories that they applied, but mostly conceptualised these theories into variables to test for associations. In addition, most of the studies included in this review applied the cross-sectional study design that could not fully accommodate dynamic theoretical propositions that capture the notion of adherence to ART or make inferences on the causality of effect [46]. These limitations were minimised by returning to the original theories to understand their explanatory power in relation to adherence to ART.

\section{Conclusion}

The focus of the review was to explore the link between behaviour change theories (or models) and mechanisms that operate during the propagation of ART adherence behaviours. We acknowledge that most social and cognitive (psychological) theories identified in this review were not formulated with the realist perspective of generative causality in mind. This is because these theories are presented in conceptual or sensitising schemes (causal models), whereby individual behaviours are explained based on various individual and environmental 'determinants'. Nevertheless, these theories offered possible underlying social drivers of behaviour that could be used to explain adherence behaviour to ART and 
other chronic medications using generative causality (explanatory theory). The candidate mechanisms and possible explanations that we have obtained from this study have implications for eliciting the programme theory of a group-based adherence intervention. Eliciting the initial programme theory entails synthesising the assumptions and perspectives of the adherence club programme designers and managers and the review of how other group-based intervention work, with the candidate mechanisms and possible explanations of ART adherence behaviour obtained from this study.

\section{Additional files}

Additional file 1: Data extraction process. This table describes the characteristics and findings of the various studies included in the review. (DOCX $54 \mathrm{~kb}$ )

Additional file 2: Data code manual. This is the coding manual that was used to indentify the various aspects of the context-mechanismoutcome heuristic tool. (DOCX $12 \mathrm{~kb}$ )

\section{Abbreviations \\ AIDS: Acquired Immune Deficiency Syndrome; ART: Antiretroviral therapy; CMO: Context-Mechanism-Outcome; HBM: Health Belief Model; HIV: Human Immuno-virus; ICAMO: Intervention-Context-Actor-Mechanism-Outcome; IMB: Information-Motivation-Behaviour; PLWHA: People Living with HIV and AIDS; PRISMA: Systematic Reviews and Meta-Analyses; SAT: Social Action Theory; SMS: Short Message Services}

\section{Acknowledgements}

We would like to acknowledge Mr Ivo N. Azia for helping us with the searching of the articles and the screening process.

\section{Funding}

The PhD from which this study emanated was funded by the Medical Research Council of South Africa in terms of the National Health Scholars Programme from funds provided for this purpose by the National Department of Health. This research was partially funded by an African Doctoral Dissertation Research Fellowship (ADDRF) award offered by the Africa Population and Health Research Center (APHRC) in partnership with the International Development Research Centre (IDRC).

\section{Availability of data and materials}

All data generated or analysed during this study are included in this published article [and its supplementary information files].

\section{Authors' contributions}

The study was conceived by FCM and BM. It was conceptualised by FCM, SVB, $\mathrm{BM}$ and BW. All the authors contributed to the development of the methodology of this study. FCM conducted the review and wrote the first draft of the present manuscript. All authors reviewed and provided comments to improve the manuscript. They also read and approved the final manuscript.

\section{Competing interests}

The authors declare that they have no competing interests.

\section{Consent for publication}

Not applicable.

\section{Ethics approval and consent to participate}

We followed the relevant standards of utility, usefulness, feasibility, propriety, accuracy and accountability while conduction the review [67].

\section{Publisher's Note}

Springer Nature remains neutral with regard to jurisdictional claims in published maps and institutional affiliations.

\section{Author details}

${ }^{1}$ School of Public Health, University of the Western Cape, Cape Town, South Africa. ${ }^{2}$ Department of Public Health, Institute of Tropical Medicine, Antwerp, Belgium. ${ }^{3}$ Institute of Development and Management, University of Antwerp, Antwerp, Belgium.

Received: 30 November 2016 Accepted: 27 April 2017

Published online: 04 May 2017

References

1. UNAIDS. 90-90-90: an ambitious treatment target to help end the AIDS epidemic. 2014; http://www.unaids.org/sites/default/files/media_asset/90-9090_en_0.pdf. Accessed 8 Feb 2017.

2. Ford N, Vitoria M, Hirnschall G, Doherty M. Getting to zero HIV deaths: progress, challenges and ways forward. J Int AIDS Soc. 2013;16:22-4. doi:10. 7448/IAS.16.1.18927.

3. Wouters E, Damme W, van Rensburg D, Meulemans H. Impact of baseline health and community support on antiretroviral treatment outcomes in HIV patients in South Africa. AIDS. 2008;22:2545-8. doi:10.1097/QAD. Ob013e32831c5562.

4. Stricker S, Fox K, Baggaley R, Negussie E, de Pee S, Grede N, et al. Retention in care and adherence to ART are critical elements of HIV care interventions. AIDS Behav. 2014;18:465-75. doi:10.1007/s10461-013-0598-6.

5. Levitt NS, Steyn K, Dave J, Bradshaw D. Chronic noncommunicable diseases and HIV-AIDS on a collision course: relevance for health care delivery, particularly in low-resource settings - insights from South Africa. Am J Clin Nutr. 2011;94(6):1690S-6S. doi:10.3945/ajcn.111.019075.

6. Bangsberg DR, Kroetz DL, Deeks SG. Adherence-resistance relationships to combination HIV antiretroviral therapy. Curr HIV/AIDS Rep. 2007:4:65-72.

7. Belayihun B, Negus R. Antiretroviral Treatment Adherence Rate and Associated Factors among People Living with HIV in Dubti Hospital, Afar Regional State, East Ethiopia. Int Sch Res Notices. 2015; 9. doi: 10.1155/ 2015/187360

8. Bangsberg DR. Less than $95 \%$ adherence to Nonnucleoside reversetranscriptase inhibitor therapy can lead to viral suppression. Clin Infect Dis. 2006;43(7):939-41.

9. Azia IN, Mukumbang FC, Van Wyk B. Barriers to adherence to antiretroviral treatment in a regional hospital in Vredenburg, Western Cape, South Africa. South Afr J HIV Med. 2016; 17(1). doi: 10.4102/sajhivmed.v17i1.476.

10. Sahay S, Reddy KS, Dhayarkar S. Optimizing adherence to antiretroviral therapy. Indian J Med Res. 2011;134(6):835-49. doi:10.4103/0971-5916.92629.

11. Patel A, Hirschhorn L, Fullem A, Ojikutu B, Oser R. Adult adherence to treatment and retention in care. 2010. http://pdf.usaid.gov/pdf_docs/ PNADX307.pdf. Accessed 8 Feb 2017.

12. World Health Organization. Retention in HIV programmes: defining the challenges and identifying solutions. Geneva: WHO Library Cataloguing-inPublication Data; 2012. http://apps.who.int/iris/handle/10665/44878. Accessed 8 Feb 2017.

13. Geng E, Glidden D, Bwana M, Musinguzi N, Emenyonu N, Muyindike W, et al. Retention in care and connection to care among HIV-infected patients on antiretroviral therapy in Africa: estimation via a sampling-based approach. PLoS One. 2011;6(7):e21797. doi:10.1371/journal.pone.0021797.

14. Boyles TH, Wilkinson LS, Leisegang $R$, et al. Factors influencing retention in care after starting antiretroviral therapy in a Rural South African Programme. PLoS One. 2011;6(5):e19201. doi:10.1371/journal.pone.0019201.

15. Lifson A, Demissie W, Tadesse A, Ketema K, May R, Yakob B, et al. Barriers to retention in care as perceived by persons living with HIV in Rural Ethiopia: focus group results and recommended strategies. J Int Assoc Provid AIDS Care. 2013;12(1):32-8. doi:10.1177/1545109712456428.

16. Bezabhe WM, Chalmers L, Bereznicki L, Peterson G, Bimirew M, Kassie $D$. Barriers and facilitators of adherence to antiretroviral drug therapy and retention in care among Adult HIV-positive patients: a qualitative study from Ethiopia. PLoS One. 2014;9(5):e97353. doi:10.1371/journal. pone.0097353.

17. Simoni J, Amico K, Pearson C, Malow R. Strategies for promoting adherence to antiretroviral therapy: a review of the literature. Curr Infect Dis Rep. 2008; 10(6):515-21. 
18. Simoni J, Amico K, Smith L, Nelson K. Antiretroviral adherence interventions: translating research findings to the real world clinic. Curr HIV/AIDS Rep. 2010;7(1):44-51. doi:10.1007/s11904-009-0037-5.

19. Joint United Nations Programme on HIV/AIDS, Médecins Sans Frontières. Community-based antiretroviral therapy delivery. Joint United Nations Programme on HIV/AIDS; 2015. http://www.unaids.org/sites/default/files/ media_asset/20150420_MSF_UNAIDS_JC2707.pdf. Accessed 8 Feb 2017.

20. World Health Organization. Adherence to long-term therapies: evidence for action. World Health Organization; 2003. http://www.who.int/chp/ knowledge/publications/adherence_introduction.pdf. Accessed 8 Feb 2017.

21. Wilkinson LS. ART adherence clubs: A long-term retention strategy for clinically stable patients receiving antiretroviral therapy. South Afr J HIV Med; 14. doi: 10.4102/hivmed.v14i2.77.

22. Rasschaert F, Decroo T, Remartinez D, Telfer B, Lessitala F, Biot M, et al. Adapting a community-based ART delivery model to the patients' needs: a mixed methods research in Tete. Mozambique BMC Public Health. 2014; 14(1):364. doi:10.1186/1471-2458-14-364.

23. Vandendyck M, Motsamai M, Mubanga M, Makhakhe S, Tunggal S, Jonckheree $\mathrm{S}$, et al. Community-based ART resulted in excellent retention and can leverage community empowerment in Rural Lesotho, a mixed method study. HIV/AIDS Res Treat Open J. 2015;2(2):44-50. doi:10.17140/ HARTOJ-2-107.

24. Khabala KB, Edwards JK, Baruani B, Sirengo M, Musembi P, Kosgei RJ, et al. Medication adherence clubs: a potential solution to managing large numbers of stable patients with multiple chronic diseases in informal settlements. Tropical Med Int Health. 2015;20(10):1265-70. doi:10.1111/tmi.12539.

25. Grimsrud A, Sharp J, Kalombo C, Bekker L-G, Myer L. Implementation of community-based adherence clubs for stable antiretroviral therapy patients in Cape Town, South Africa. J Int Aids Soc. 2015;18(1). doi: 10.7448/IAS.18.1. 19984.

26. Luque-Fernandez MA, Van Cutsem G, Goemaere E, Hilderbrand K, Schomaker M, Mantangana N, et al. Effectiveness of patient adherence groups as a model of care for stable patients on antiretroviral therapy in Khayelitsha, Cape Town, South Africa. PLoS One. 2013;8(2):e56088. doi:10. 1371/journal.pone.0056088.

27. Luque-Fernandez MA, Van Cutsem G, Goemaere E, Hilderbrand K, Schomaker M, Mantangana N, et al. Community-based antiretroviral therapy programs can overcome barriers to retention of patients and decongest health services in sub- saharan africa: a systematic review. Int Health. 2013; 5(3):169-79. doi:10.1093/inthealth/iht016.

28. Chaiyachati $\mathrm{KH}$, Ogbuoji O, Price M, Suthar AB. Interventions to improve adherence to antiretroviral therapy: a rapid systematic review. AIDS. 2014; 28(Suppl 2):S187-204. doi:10.1097/QAD.0000000000000252.

29. Hall BJ, Sou K-L, Beanland R, Lacky M, Tso LS, Ma Q, et al. Barriers and facilitators to interventions improving retention in HIV care: a qualitative evidence meta-synthesis. AIDS Behav. 2016; doi:10.1007/s10461-016-1537-0.

30. Ma Q, Tso LS, Rich ZC, Hall BJ, Beanland R. Li H; barriers and facilitators of interventions for improving antiretroviral therapy adherence: a systematic review of global qualitative evidence. J Int AIDS Soc. 2016;19(1):21166. http://doi.org/10.7448/IAS.19.1.21166.

31. Wouters E, Damme W, van Rensburg D, Masquillier C, Meulemans H. Impact of community-based support services on antiretroviral treatment programme delivery and outcomes in resource-limited countries: a synthetic review. BMC Health Serv Res. 2012;12(1):194. doi:10.1186/1472-6963-12-194.

32. Funnell SC. Developing and using a program theory matrix for program evaluation and performance monitoring. N Dir Eval. 2000;87(1):91-101. doi: 10.1002/ev.1185.

33. Funnell SC, Rogers PJ. Purposeful program theory: Effective use of theories of change and logic models. San Francisco: Wiley; 2011.

34. Mukumbang FC, Van Belle S, Marchal B, van Wyk B. A realist evaluation of the antiretroviral treatment adherence club programme in selected primary health care facilities in the metropolitan area of Western Cape Province, South Africa: a study protocol. BMJ Open. 2016;6:e009977. doi:10.1136/ bmjopen-2015009977

35. Mukumbang FC, Van Belle S, Marchal B, van Wyk B. Towards developing an initial Programme theory: Programme designers and managers assumptions on the antiretroviral treatment adherence Club Programme in primary health care facilities in the metropolitan area of Western Cape Province, South Africa. PLoS One. 2016;11(8):e0161790. doi:10.1371/journal.pone.0161790.

36. Pawson R, Tilley N. Realistic evaluation. 2nd ed. London: SAGE Publications; 1997.
37. Westhorp G. Using complexity-consistent theory for evaluating complex systems. Evaluation. 2012;18(4):405-20. doi:10.1177/1356389012460963.

38. Lacouture A, Breton E, Guichard A, Ridde V. The concept of mechanism from a realist approach: a scoping review to facilitate its operationalization in public health program evaluation. Implement Sci. 2015;10(1):153. doi:10. 1186/s13012-015-0345-7.

39. Pedersen $L$, Rieper $O$. Is realist evaluation a realistic approach for complex reforms? Evaluation. 2008;14(3):271-93. doi:10.1177/1356389008090856.

40. Pawson R, Tilley N. Realist Evaluation. British Cabinet Office; 2004. http:// www.communitymatters.com.au/RE_chapter.pdf. Accessed 8 Feb 2017.

41. de Souza DE. Elaborating the Context-Mechanism-Outcome configuration (CMOc) in realist evaluation: a critical realist perspective. 2013; 19(2):141-154 doi: 10.1177/1356389013485194.

42. Van Belle SB. Accountability in sexual and reproductive health how relations between INGOs and state actors shape public accountability: a study of two local health systems in Ghana. United Kingdom: London School of Hygiene and Tropical Medicine University of London; 2014. p. 1-382.

43. Arksey H, O'Malley L. Scoping studies: towards a methodological framework. Int J Soc Res Methodol. 2005:8(1):19-32. doi:10.1080/1364557032000119616.

44. Dijkers M. What is a Scoping Review? KT Update. 2015; 4(1). http://ktdrr.org/ products/update/v4n1/dijkers_ktupdate_v4n1_12-15.pdf. Accessed 8 Feb 2017.

45. Torraco RJ. Writing integrative literature reviews: guidelines and examples. Hum Resour Dev Rev. 2005;4(3):356-67. doi:10.1177/1534484305278283.

46. Holmes E, Hughes D, Morrison V. Predicting adherence to medications using Health Psychology theories: a systematic review of 20 years of empirical research. Value Heal. 2014;17(8):863-76. http://dx.doi.org/10. 1016/j.jval.2014.08.2671

47. Fisher JD, Fisher WA. Changing AIDS-risk behavior. Psychol Bull. 1992; 111:455-74

48. Ewart CK. Social action theory for a public health psychology. Am Psychol. 1991:46:931-46

49. Janz NK, Becker MH. The health belief model: a decade later. Health Educ Q. 1984;11(1):1-47.

50. Fisher J, Fisher W, Amico K, Harman J. An information-motivationbehavioral skills model of adherence to antiretroviral therapy. Health Psychol. 2006;25:462-73.

51. Fisher J, Amico K, Fisher W, Harman J. The information-motivation-behavioral skills model of antiretroviral adherence and its applications. Curr HIV/AIDS Rep. 2008;5:193-203.

52. Amico KR, Barta W, Konkle-Parker DJ, et al. The information-motivationbehavioral skills model of ART adherence in a deep South HIV+ clinic sample. AIDS Behav. 2009;13:66-75.

53. Amico KR, Toro-Alfonso J, Fisher JD. An empirical test of the information, motivation and behavioral skills model of antiretroviral therapy adherence. AIDS Care. 2005;17:661-73.

54. Starace F, Massa A, Amico K, Fisher J. Adherence to antiretroviral therapy: an empirical test of the information-motivation-behavioral skills model. Health Psychol. 2006;25:153-62

55. Horvath KJ, Smolenski D, Amico KR. An empirical test of the informationmotivation-behavioral skills model of ART adherence in a sample of HIVpositive persons primarily in out-of-HIV-care settings. AIDS Care. 2014;26: 142-51. doi:10.1080/09540121.2013.802283.

56. Fisher J, Cornman D, Norton W, Fisher W. Involving behavioral scientists, health care providers, and HIV-infected patients as collaborators in theorybased HIV prevention and antiretroviral adherence interventions. J Acquir Immune Defic Syndr. 2006:43(Suppl 1):S10-7.

57. Norton W, Amico K, Fisher W, Shuper P, Ferrer R, Cornman D, et al. Information-motivation-behavioral skills barriers associated with intentional versus unintentional ARV non-adherence behavior among HIV+ patients in clinical care. AIDS Care. 2010;22(8):979-87. doi:10.1080/09540121003758630

58. Torija CST, Vázquez VG, Montijo SSR, Romo LE. The information and motivation and behavioral skills model of ART adherence among HIVpositive adults in Mexico. J Int Assoc Provid AIDS Care. 2015;14(4):335-42. doi:10.1177/2325957415581903

59. Kalichman SC, Kalichman MO, Cherry C, Swetzes C, Amaral CM, White D, et al. Brief behavioral self-regulation counseling for HIV treatment adherence delivered by cell phone: an initial test of concept trial. AIDS Patient Care STDs. 2011;25(5):303-10. http://doi.org/10.1089/apc.2010.0367.

60. Munro S, Lewin S, Swart T. A review of health behaviour theories: how useful are these for developing interventions to promote long-term 
medication adherence for TB and HIV/AIDS? BMC Public Health. 2007; 7(104). doi: 10.1186/1471-2458-7-104.

61. Johnson MO, Catz SL, Remien RH, Rotheram-Borus MJ, Morin SF, Charlebois $\mathrm{E}$, et al. Theory-guided, empirically supported avenues for intervention on HIV medication nonadherence: findings from the healthy living project. AIDS Patient Care STDs. 2003;17(12):645-56.

62. Remien R, Stirratt M, Dognin J, Day E, El-Bassel N, Warne P. Moving from theory to research to practice: implementing an effective dyadic intervention to improve antiretroviral adherence for clinic patients. J Acquir Immune Defic Syndr. 2006;43:S69-78.

63. Conner M, Norman P. Predicting health behaviour: a social cognition approach. Second ed. London: Open University Press; 2005. p. 1-27.

64. Becker MH, Maiman LA, Kirscht JP, Haefner DP. The health belief model and prediction of dietary compliance: a field experiment. J Health Soc Behav. 1977;18(4):348-66.

65. Krueger KP, Berger BA, Felkey B. Medication adherence and persistence: a comprehensive review. Adv Ther. 2005;22(4):313-56.

66. Bandura A. Social cognitive theory: an agentic perspective. Annu Rev Psychol. 2001;1(52):1-26.

67. Pawson R, Boaz A, Grayson L, Long A, Barnes C. Types and quality of social care knowledge Stage two: towards the quality assessment of social care knowledge. ESRC UK Centre for Evidence Based Policy and Practice: Working Paper 18. https:/www.kcl.ac.uk/sspp/departments/politicaleconomy/research/ cep/pubs/papers/assets/wp18.pdf. Accessed 8 Feb 2017.

\section{Submit your next manuscript to BioMed Central} and we will help you at every step:

- We accept pre-submission inquiries

- Our selector tool helps you to find the most relevant journal

- We provide round the clock customer support

- Convenient online submission

- Thorough peer review

- Inclusion in PubMed and all major indexing services

- Maximum visibility for your research

Submit your manuscript at www.biomedcentral.com/submit 